trum, external capsule, putamen and head of the caudate nucleus. There was no evidence of cortical abnormality.

She was treated with clonazepam, pirazepam, madopar and bromocriptine, in various combinations, but she did not respond to treatment.

On a neuropsychological assessment, the patient was cooperative and well oriented. She complained of depression and difficulties with memory. The WAIS results were within the average range (Verbal IQ 104; Performance IQ 99). The Wechsler memory IQ was Low Average (MQ89). There was, however, some discrepancy on the results of the memory test. For instance, she recalled $9 / 24$ items of information in one of the prose passages, but only 4/23 in another. She had a poor digit span (four digits forwards, two digits backwards). Her score on paired associates was also poor. She scored $3 / 10$ on the first trial, and she did not improve with further presentations. On the other hand her ability to recall visual material was good and she made no errors on immediate recall. There was no evidence of specific language difficulties or other left hemisphere symptoms such as acalculia or constructional apraxia.

Because of her low productivity at work, and the fact that apraxia has been reported in patients with subcortical damage,${ }^{4}$ the patient was assessed with tests of motor skill and dyspraxia. She was slow, clumsy and lacked accuracy on three tests of motor skill requiring speed and accuracy of hand movements (Reitan-trial-making test, tracking test and tapping test). When performing these tests, she displayed a fine tremor of the left hand, which was not apparent on neurological examination. She was also asked to perform hand movement sequences and alternating movements of the left and right hand; her performance was distinctly inaccurate, and she was unable to improve after several trials.

Praxis evaluation was based on the performance of 20 meaningful gestures (for example, waving goodbye, using a comb) to command and imitation. She was also asked to imitate 10 meaningless hand/arm positions demonstrated by the examiner. In addition, she was asked to handle 10 single objects, and to perform more complex actions-on command and imitation-which required handling more than one object. She was also asked

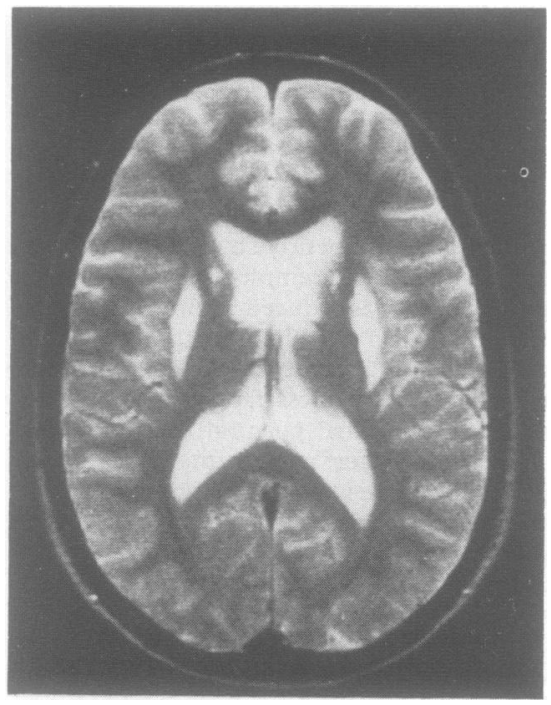

Figure Axial SE T2 weighted (TR $2400 \mathrm{~ms} / T E 90 \mathrm{~ms}$ ) image through mid lateral ventricular level ( see text). to perform 10 facial gestures on command and imitation. Her performance on the tests requiring arm/hand movements was poor and she made a number of errors similar to those seen in patients with apraxia.

She was unable to perform contralateral movements on imitation of meaningless postures. For instance, when asked to imitate a posture that required her to touch her left ear with the right hand, she touched her right ear with the right hand. She also made dyspraxic errors when handling real objects. Thus she took the comb by its teeth and attempted to comb her hair using the wrong side. She also attempted to strike a match with the wrong end. There was no evidence of oral apraxia.

This patient showed mild Parkinsonian features and, on tests of motor skill, she was slow, clumsy and inaccurate. In addition, there was a praxic impairment. The presence of praxic problems confirms the view that apraxia, usually considered a cortical sign, may also occur with lesions in the sub-cortical structures. This case is also interesting because the praxic problems did not result from an abrupt vascular accident, as in most previously reported cases.

Our findings support the view of De Renzi et $\mathrm{al}^{4}$ that the classical cortical lesions causing apraxia must be enlarged to encompass a basal ganglia-thalamus-cortex loop.

M J MOZAZ Faculty of Psychology, University of the Basque Country, San Sebastian, Spain Psychology Department Institute of Psychiatry London, UK B INDAKOETXEA Section of Neurology, Hospital Ntra Sra de Aranzazu, San Sebastian, Spain

1 Guggenheim MA, Couch JR, Weinberg W. Motor dysfunction as a permanent complicaMotor dysfunction as a permanent complication of menth

2 McLean DR, Jacobs H, Mielke BW. Methanol poisoning: a clinical and pathological study. Ann Neurol 1980;8:161-7.

3 Oliveras LC, Galindo GF. Parkinsonian syndrome after methanol intoxication. Eur Neuro 1983;22:405-9.

4 De Renzi E, Faglioni P, Scarpa M, Crisi G Limb apraxia in patient with damage confined to the left basal ganglia and thalamus. $J$ Neurol Neurosurg Psychiatry 1986;49:1030-8.

\section{Botulism with sensory symptoms: a second case}

In 1982 Goode and Shearn described a case of botulism with pinprick decreased sensation in the territory of the left trigeminal nerve. ${ }^{\prime}$ Our patient with botulism had left hemianalgesia of the body and the face.

A 43 year old woman developed vomiting and abdominal discomfort, with blurred vision, diplopia, dysphagia, dysphonia, dry mouth and numbness on the left side of her body and her face. She also had constipation and urine retention, that required bladder catheterisation. Six days before admission, she had ingested home-canned green beans. Her past medical history was unremarkable.

On neurological examination there were bilateral nonreactive mydriasis, ophthalmoplegia and ptosis, weakness of the soft palate and tongue with impairment of pharyngeal reflexes. She presented a decreased pinprick sensation on the left half of her body and her face. She had a mild diffuse global weakness. Reflexes were brisk and plantar responses flexor. Her skin and her mouth were dry. A Schirmer test was positive. A CT scan of the head and a CSF analysis were normal. Motor and sensory conduction velocities of the right ulnar nerve were normal. The $\mathrm{M}$-wave amplitudes were small $(1-3 \mathrm{mV})$ at sporadic stimulation, without any change at $2 \mathrm{~Hz}$ of repetitive stimulation, but at $30 \mathrm{~Hz}$ there was an increase of $183 \%$. There was also a moderate dysfunction of the autonomic nervous system assessed by standard tests. ${ }^{23}$ Blood pressure fell $24 \mathrm{~mm} \mathrm{Hg}$ (systolic) and $5 \mathrm{~mm} \mathrm{Hg}$ diastolic on standing. Heart rate response to standing: the ratio of the $R-R$ interval at beat 30 after standing to the $R-R$ interval at beat 15 after standing ${ }^{23}$ was 0.9 . Isometric exercise: the increase of diastolic $\mathrm{BP}$ was $6 \mathrm{~mm} \mathrm{Hg}$. Heart rate variation with respiration: the expiration/inspiration ratio was $1 \cdot 03$. The Valsalva ratio was $1 \cdot 05$. A serum specimen and a sample of the contaminated food were positive for type B botulinum toxin.

The patient's condition started to improve after five days. The left hemianalgesia disappeared on the sixth day and the hyperreflexia on the seventh day. She received botulinum trivalent antitoxin for three days after admission. Eighteen days later she developed a right acute suppurative parotitis, which resolved with antimicrobials and antiinflammatory agents. At discharge, one month after admission, she complained of a dry mouth and dry eyes. Two months later she was asymptomatic.

The clinical, neurophysiological ${ }^{4}$ and laboratory findings were consistent with a diagnosis of botulism, but the presence of sensory signs surprised the neurologists who examined the patient. The possibility of a stroke seemed unlikely since the patient had no risk factors, the symptoms developed concomitantly with the evolution of botulism, and a CT scan of the head was normal. The acute suppurative parotitis may have been related to a reduced production of saliva.

Botulinum toxin causes a marked reduction in the number of quanta released by a nerve stimulus. In vitro, at low doses, it blocks only cholinergic transmission and preferentially motor end-plates, but at high doses it may block other types of transmission. ${ }^{5}$

In the case reported by Goode and Shearn, the patient presented a hyperaesthesia on the left trigeminal territory, and they suggested that a multiple mononeuropathy could be underlying the clinical findings. Tendon reflexes were brisk, as was the case in our patient, a feature which is considered unusual in botulism.

Our patient showed impairment of neuromuscular and autonomic transmission. Only an impaired central transmission could probably explain her left pinprick hemihyperaesthesia, and therefore the possible presence of botulinum toxin within the CNS Most authors believe that a normal bloodbrain barrier function prevents botulinum toxin entering the brain tissue, but intraaxonal transport of radiolabelled toxin has been proved. ${ }^{6}$ Although we have no satisfactory explanation for sensory symptoms in our patient, we conclude, as do Goode and Shearn, that sensory abnormalities and 
hyperreflexia would not exclude a diagnosis of botulism.

\section{JC MARTÍNEZ-CASTRILLO MA DEL REAL
A HERNANDEZ GONZALEZ G DE BLAS * \\ JC ALVAREZ-CERMEÑO
Servicio de Neurologia, Servicio de Neurologia, \\ Servicio de Neurofisiologia,
Hospital Ramón y Cajal, 28034 Madrid, Spain}

Correspondence to Dr Martinez Castrillo.

1 Goode GB, Shearn DL. Botulism. A case with associated sensory abnormalities. Arch Neurol 1982;39:55.

2 McLeod JG, Tuck RR. Disorders of the autonomic nervous system. Part 2: Investigation nomic nervous system. Part 2: Investigation

3 Vita G, Girlanda P, Puglisi RM, Marabello I Messina C. Cardiovascular-reflex testing and single-fiber electromyography in botulism. A longitudinal study. Arch Neurol 1987;44: 202-6.

4 Pickett JB. Botulism. Muscle Nerve 1988;11:1201-5.

5 Bigalke H, Dreyer F, Bergey G. Botulinum A neurotoxin inhibit non-cholinergic synaptic transmission in mouse spinal cord neurons in culture. Brain Res 1985;360:318-24.

6 Sugiyama H. Clostridium botulinum neurotoxin. Microbiol Rev 1980;44:419-48.

\section{Herpes simplex encephalitis following a skull fracture}

Herpes simplex encephalitis is an uncommon and severe infection, in which early detection and treatment significantly improve the outlook. ' We report a case following a depressed skull fracture.

A nine year old boy with diabetes since the age of two was admitted with a head injury following a fall from his bicycle. Examination revealed a right frontal laceration. Skull radiographs showed an extensive bifrontal fracture, depressed on the left. At operation brain and cerebrospinal fluid was seen oozing from the laceration. A bicoronal scalp flap was turned. The depressed area was elevated and cleaned, and a burr hole was made to aid exposure and repair of a dural tear on the right. Ampicillin and flucloxacillin were given intravenously for three days and subsequently orally for four more days. He was discharged on day 5 with no neurological deficit, and was given sodium valproate prophylactically.

On day 20 he was readmitted with a two day history of moodiness and staring episodes culminating in a generalised convulsion. Neurological examination was normal and he was apyrexial. Blood sugar was $8 \mathrm{mmol} / 1$. Diazepam was given rectally when a generalised convulsion was observed on the ward. A diagnosis of post traumatic epilepsy was made. An electroencephalogram showed moderate amplitude irregular waves in the frontal leads compatible with the recent injury. By day 23 he had had further episodes preceded by sensations of odd tastes and manifesting as altered consciousness with jaw clenching, drooling and post-ictal left sided weakness. Carbamazepine was added, and the frequency of these episodes decreased. On day 25 a cranial CT scan was normal.

On day 27 he developed a pyrexia and right sided earache. Examination of the ear was normal. The fever recurred the following day, and intermittent seizures progressed to focal status epilepticus which was controlled with an infusion of chlormethiazole. Lumbar puncture revealed 38 white cells, predominantly lymphocytes, and one red cell per high power field. CT scan showed an area of mixed density with enhancement in the right temporal region (figure). This raised the possibility of either an evolving bacterial abscess (although the CT scan lesion was contralateral from the site of the depressed part of the fracture), or a focal encephalitis. He was accordingly treated with chloramphenicol $100 \mathrm{mg} / \mathrm{kg} /$ day, benzylpenicillin $200 \mathrm{mg} / \mathrm{kg}$ day, metronidazole $22 \mathrm{mg} / \mathrm{kg} /$ day, and acyclovir $10 \mathrm{mg} / \mathrm{kg} /$ day. On day 29 he deteriorated with diabetic ketoacidosis. A small ulcer was seen on his lip. A further electroencephalogram showed diffuse severe abnormality, with very high amplitude irregular delta waves occurring in all leads. Despite his increasingly critical condition, a right craniotomy was performed on day 31 to exclude the possibility of an abscess, and this revealed soft inflamed brain with no abscess. Histology of the biopsy specimen showed cerebral cortex containing foci of necrosis with oedema. Perivascular cuffing with lymphocytes was observed; occasional polymorphs and eosinophilic cells were seen. Gram stain for microorganisms was negative.
Herpes simplex virus was identified on electron microscopy, and was also subsequently cultured from the biopsy specimen. He was given ventilation for four days postoperatively. Acyclovir was continued for 10 days. Recovery was complicated by a dense left hemiparesis and a pseudobulbar palsy. Subsequently, he made an impressive recovery, and is currently able to run, but has dysarthria, and has little use in his left hand.

Intracerebral bacterial infection is a well recognised but, with appropriate management, a relatively uncommon complication of compound skull fractures, occurring at a rate of $4 \%$ in one large series. ${ }^{2}$ There are no previous reports of herpes simplex encephalitis (HSE) following a skull fracture. Contamination of the wound is the route of spread of bacterial infection, but our case presumably represents a reactivated latent infection (from viral deoxyribonucleic acid present in the brain) in response to the considerable stress of the injury, rather than innoculation during the original injury.

Absence of a fever and an initially normal CT scan delayed the identification of infection as the cause until 10 days after the onset of symptoms, during which time the diagnosis was of post traumatic epilepsy. Craniotomy and brain biopsy are not felt to be mandatory to reach a diagnosis of HSE in children in whom there is little to suggest an alternative diagnosis, ${ }^{3}$ but in this case it was felt important to exclude the possibility of a cerebral abscess.

Focal seizures with altered consciousness should always alert the clinician to the possibility of HSE, even in the absence of a fever or CT scan changes.

\section{P D CAMERON P BLACKBURN S J WALLACE Department of Paediatrics, University Hospital of Wales, Heath Park, Cardiff and Department of Neurosurgery, The Hospital for Sick Children,
Great Ormond Street, London}

1 Whitley RJ, Alford CA, Hirsch MS. Vidarabine versus acyclovir therapy in herpes simplex encephalitis. N Engl J Med 1986;314:144-9.

2 Sande GM, Galbraith SL, McLatchie G. Infection after depressed fracture in the west of Scotland. Scot Med J 1980;25:227-9.

3 Brett EM. Herpes simplex encephalitis in children. $B M J$ 1986;293:1388-9.

Could lamotrigine be useful in status epilepticus? A case report

Lamotrigine, a new putative antiepileptic drug ${ }^{1}$ may have been effective in a case of status epilepticus. The patient was a 17 year old, mentally handicapped, right handed girl who had experienced epilepsy of unknown aetiology from the age of nine months and had been attending our clinic since 1981 . At the time of her latest admission to hospital, the patient had been taking a combination of carbamazepine and phenobarbitone at daily doses of 1200 and $200 \mathrm{mg}$, respectively for about a year, with partial control of seizures (nocturnal tonic fits: $10-30$ per month; atypical absences: 3-5/month; atonic seizures: 3-5/month). Interictal EEG recording was characterised by diffuse slow waves, occasionally accompanied by spikes or polyspikes isolated or grouped in symmetrical, generalised bursts. She was admitted to hospital for an unexplained sudden increase in tonic seizures (up to 4-6/hour without recovery of consciousness between fits). A 